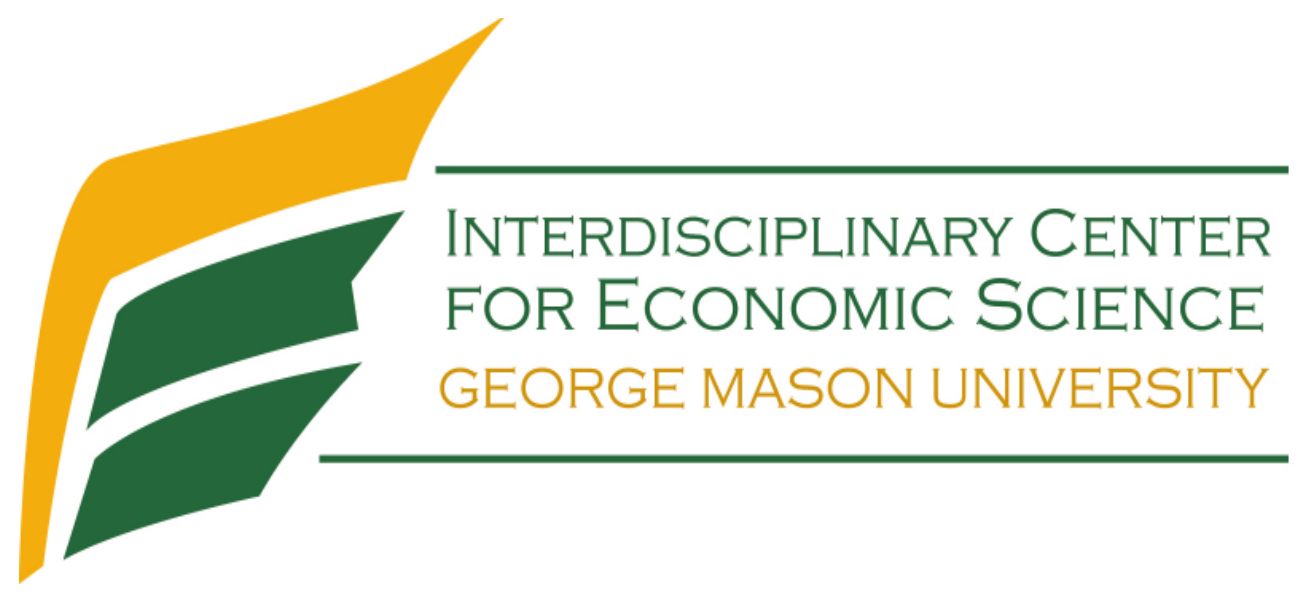

\title{
An Algebraic Approach to Revealed Preference
}

Mikhail Freer and César Martinelli

May 2020

Discussion Paper

Interdisciplinary Center for Economic Science

4400 University Drive, MSN 1B2, Fairfax, VA 22030

Tel: +1-703-993-4850 Fax: +1-703-993-4851

ICES Website: http://ices.gmu.edu

ICES RePEc Archive Online at:http://edirc.repec.org/data/icgmuus.html 


\title{
AN ALGEBRAIC APPROACH TO REVEALED PREFERENCE
}

\author{
MIKHAIL FREER \\ University of Essex \\ CÉSAR MARTINELLI \\ George Mason University
}

\begin{abstract}
We propose and develop an algebraic approach to revealed preference. Our approach dispenses with non algebraic structure, such as topological assumptions. We provide an algebraic axiom of revealed preference that subsumes previous, classical revealed preference axioms, and show that a dataset is rationalizable if and only if it is consistent with the axiom.
\end{abstract}

\section{INTRODUCTION}

The revealed preference approach to consumer choice, pioneered by Samuelson (1938), builds on the fact that, although we cannot observe the complete preference relation profiles of economic agents, we can observe their choices over some budget sets. Starting with the work Richter (1966) and Afriat (1967), this approach has been used to construct tests of rational decision making (see Chambers and Echenique, 2016, for a recent comprehensive overview).

E-mail addresses: (Freer) m.freer@essex.ac.uk, (Martinelli) cmarti33@gmu .edu.

A previous version of this paper was circulated under the title "A Functional Approach to Revealed Preferences." We gratefully acknowledge Marco Castillo, Christopher Chambers, Thomas Demuynck, Federico Echenique, and Matthew Pollison for useful comments. 
Contribution. We propose an algebraic version of revealed preference approach. That is, we consider theories about preferences in their logical structure together with the underlying algebraic structure. By a theory about preferences, we mean a statement about preferences such as "If $x$ is better than $y$ then $f(x)$ better than $f(y)$, where $f \in \mathcal{F}$." In this statement, $f$ is some function over alternatives and $\mathcal{F}$ is a family of functions that actually defines the theory. The algebraic structure we impose considers the algebra of $(\mathcal{F}, \circ)$, where $\circ$ is the composition operator. In particular, we propose an algebraic axiom of revealed preferences and show that if $(\mathcal{F}, \circ)$ is a group, ${ }^{1}$ then the observed set of data could be generated by a preference relation if and only if it is consistent with the algebraic axiom of revealed preferences. We show that our result subsumes several existing tests of revealed preference that include transitive (see Afriat (1967), Varian (1983), Forges and Minelli (2009), and Nishimura et al. (2017)), homothetic (see Varian (1983), Heufer (2013), and Heufer and Hjertstrand (2019)) and quasilinear (see Brown and Calsamiglia (2007) and Castillo and Freer (2020)) preferences.

Our approach can be considered a continuation of the research by Chambers et al. (2014). A major result of that paper is that, if a theory about preferences can be axiomatized with so-called "universal negation of conjunctions of atomic formulae" (UNCAF), then the theory is falsifiable. We take on the next step, making explicit the algebraic structure of the theory and presenting a revealed preference axiom that is necessary and sufficient to test any of such theory. We also show that, under some extra conditions, completeness of preferences has no additional empirical content. This fact is consistent with the work of Chambers et al. (2014), since, as they explicitly mention, completeness is not an UNCAF axiom.

Other related literature. Our work is linked to the literature on generalized revealed preferences. Several authors in this literature provide a generalization of the revealed preference approach, but keeping some

\footnotetext{
${ }^{1}$ A tuple $(\mathcal{F}, \circ)$ is said to be group if the set of functions $\mathcal{F}$ contains an identify function, $\mathcal{F}$ is closed, every function in $\mathcal{F}$ has an inverse that also belongs to $\mathcal{F}$, and the composition operator $\circ$ is associative.
} 
topological assumptions in place. Topological assumptions are necessary to guarantee existence of a convenient utility function representing the underlying preference relation. Seminal examples are Forges and Minelli (2009) and Nishimura et al. (2017), who generalize Afriat (1967) theorem for general shapes of budgets and topological spaces. Recently, Polisson, Quah, and Renou (2015) have proposed a lattice approach, and provided conditions that guarantee the rationalization of an observed set of data with theories such as expected utility, ranked dependent expected utility, and cumulative prospect theory. The papers mentioned above construct tests which can be easily applied to the data.

Other authors generalize the Szpilrajn (1930) result concentrating on the completion of the revealed preference relation. Seminal papers by Suzumura (1976), Duggan (1999), and Demuynck (2009) provide revealed preference tests (in the shape of Suzumura consistency) for transitive, acyclic, homothetic, and convex preferences. However, the Suzumura consistency condition may be complicated for practical implementation, which is an important difference with the papers mentioned in the previous paragraph. In this sense, we provide a link between these two strands of revealed preference research. That is, we adopt a scope of theories comparable to one presented in Demuynck (2009), while providing a tractable and simple revealed preference axiom.

Organization of the paper. The remainder of the paper is organized as follows. We present the necessary definitions for algebraic revealed preferences in section 2 . We show our rationalizability result in section 3. We provide some concluding remarks in section 4. All proofs omitted in the text are collected in an Appendix.

\section{PRELiminaries}

Let $X$ be the space of alternatives. Let $R \subseteq X \times X$ be a preference relation, that is a reflexive binary relation; that is, $(x, x) \in R$ for every $x \in X$. Let $P(R)$ is the (asymmetric) strict part of the relation; that is, $P(R)=\{(x, y) \in R:(y, x) \notin R\}$. Denote by $\mathcal{R}$ the space of preference relations. 
2.1. Theories about preferences. Next we present our notion of a theory about preference relations. Our notion is meant to capture the idea that a theory is a collection of desirable properties of preference relations. We first discuss some usual theories to motivate our notion.

Example: Consider a theory that imposes only transitive preferences, that is $(x, y),(y, z) \in R$ implies that $(x, z) \in R$. Equivalently, if there is a finite sequence $x=s_{1}, \ldots, s_{n}=y \in X$ such that $\left(s_{j}, s_{j+1}\right) \in$ $R$ for every $j \in\{1, \ldots, n-1\}$, then $(x, y) \in R$.

Note that transitivity imposes a particular structure over the preference relation that admits a sequential representation. That is, $(x, y) \in$ $R$ if every pair connecting $x$ and $y$ also belongs to $R$. We will generalize this structure and show that other usual properties can be imposed in the same manner.

Example: Consider a theory that imposes transitive and homothetic preferences, that is $(x, y),(y, z) \in R$ implies $(x, z) \in R$ and $(x, y) \in R$ if and only if $(\alpha x, \alpha y) \in R$ for every $\alpha \in \mathbb{R}_{++}$. Equivalently, if there are sequences $x=s_{1}, \ldots, s_{n}=y \in X$ and $\alpha_{1}, \ldots, \alpha_{n-1} \in \mathbb{R}_{++}$such that $\left(\alpha_{j} s_{j}, \alpha_{j} s_{j+1}\right) \in R$ for every $j \in\{1, \ldots, n-1\}$, then $(x, y) \in R$.

Like in the case of transitivity alone, imposing jointly transitivity and homotheticity admits a sequential representation. That is, $(x, y) \in R$ if every pair $\left(s_{j}, s_{j+1}\right)$ connecting $x$ and $y$ satisfies $\left(f_{j}\left(s_{j}\right), f_{j}\left(s_{j+1}\right)\right) \in R$ for some $f_{j} \in \mathcal{F}$, where $\mathcal{F}$ is a collection of functions satisfying $f$ : $X \rightarrow X$ for all $f \in \mathcal{F}$. In the case of transitivity and homotheticity, $\mathcal{F}$ is simply the collection of linear functions with positive slope. We generalize this idea allowing for other collections of functions, so that every allowed collection of functions $\mathcal{F}$ defines a theory.

First, we require the collection of functions $\mathcal{F}$ endowed with the composition operator to be a group.

Definition 1. A tuple $(\mathcal{F}, \circ)$ is a group if it

- contains identity: $I \in \mathcal{F}$, where $I(x)=x$ for every $x \in X$; 
- is closed:

$f, f^{\prime} \in \mathcal{F}$ implies $f \circ f^{\prime}=f^{\prime \prime} \in \mathcal{F}$;

- has inverse element:

$\forall f \in \mathcal{F} \exists f^{-1} \in \mathcal{F}$ such that $f \circ f^{-1}=f^{-1} \circ f=I$;

- is associative:

$\left(f \circ f^{\prime}\right) \circ f^{\prime \prime}=f \circ\left(f^{\prime} \circ f^{\prime \prime}\right)$ for all $f, f^{\prime}, f^{\prime \prime} \in \mathcal{F}$.

Note that the collection of linear functions with positive slope endowed with the composition operator constitutes a group. The requirement of identity means that the theories we consider impose transitivity. Closedness simply allows to consider shorter sequences $x=$ $s_{1}, \ldots, s_{n}=y \in X$ connecting $x$ and $y$. Associativity is a property of the composition operator. The requirement of inverse element is more substantial; intuitively, it means that if it is desirable that $(x, y) \in R$ implies $(f(x), f(y)) \in R$, then the reverse causality is desirable as well.

We also require that preference relations satisfy monotonicity. That is, we endow $X$ with a partial order $\geq$ with $>$ being its (asymmetric) strict part, and say a preference relation $R$ is monotone if $(x, y) \in \geq$ implies $(x, y) \in R$ and $(x, y) \in>$ implies $(x, y) \in P(R)$. Intuitively, $(X, \geq)$ is a space of goods, and $(x, y) \in>$ means that $x$ is greater and thus more desirable than $y$.

Definition 2. Let $(\mathcal{F}, \circ)$ be a group. A preference relation $R$ is consistent with theory $\mathcal{F}$ if it is monotone and for every pair of sequences $x=s_{1}, \ldots, s_{n}=y \in X$ and $f_{1}, \ldots, f_{n-1} \in \mathcal{F}$, such that

$$
\left(f_{j}\left(s_{j}\right), f_{j}\left(s_{j+1}\right)\right) \in R \text { for every } j \in\{1, \ldots, n-1\},
$$

we have $(x, y) \in R$.

Our notion of the theory is a specification of a universal negation of a conjunction of atomic formula (UNCAF) axiomatizable theory from Chambers et al. (2014). ${ }^{2}$ However, we already embed over the functions

\footnotetext{
${ }^{2}$ Our definition uses the implication operator, but we can equivalently rewrite it in the canonical UNCAF axiomatization as follows

$$
\forall s_{1} \forall s_{2} \ldots \forall s_{n}, \neg\left(\bigwedge_{j=1}^{n-1}\left(f_{j}\left(s_{j}\right), f_{j}\left(s_{j+1}\right)\right) \in R \wedge(x, y) \in N(R) \cup P\left(R^{-1}\right)\right),
$$

where $N(R)=\{(x, y):(x, y),(y, x) \notin R\}$ is the noncomparable part of the preference relation $R$, and $R^{-1}=\{(x, y):(y, x) \in R\}$ is the inverse preference relation.
} 
the algebraic structure (group) which induces the desired properties. Importantly, note that a theory is uniquely determined by the family of functions $\mathcal{F}$.

Our notion of theory does not include completeness, which is technically difficult to handle. ${ }^{3}$ Moreover, it is often the case that completeness does not have an empirical content in the revealed preference framework. We discuss this point in section 3.2.

We assume that $\geq$ is consistent with the theory $\mathcal{F}$. This is a technical, although crucial assumption. If $\geq$ is not consistent with the theory, then the problem of seeking a monotone preference relation that is consistent with $\mathcal{F}$ is nonsensical.

2.2. Data and Rationalization. The essence of the revealed preference problem is to extract the (unobserved) preference relations which generated (observed) choices over budgets. If there is such a preference relation, then the corresponding data set (collection of choices from budgets) is rationalizable. Next, we formally define the rationalizable data sets.

Let $B \subseteq X$ be a budget set, where $B$ is any nonempty subset of $X$. Let $\mathcal{B}$ be a collection of budgets. Let $C: \mathcal{B} \rightarrow X$ be a choice function. Denote by $(\mathcal{B}, C)$ a data set. All the results below holds for choice correspondences; for notational simplicity we consider them as observed via repeated singleton choices from each budget set.

Definition 3. A data set $(\mathcal{B}, C)$ is rationalizable with theory $\mathcal{F}$ if there is a preference relation $R^{*}$ consistent with theory $\mathcal{F}$ such that

$$
(x, y) \in R^{*} \text { for every } x \in C(B) ; y \in B ; B \in \mathcal{B} .
$$

The definition of rationalizability specifies that there is a preference relation consistent with a given theory, such that the observed choices can be generated by maximization of this preference relation. Note that we abstain from any restrictions on the cardinality of the data set and any topological structure of the space of alternatives, since we are

\footnotetext{
${ }^{3}$ Neither completeness nor non-satiation are UNCAF axioms, and therefore do not fit the framework (see p. 2305 in Chambers et al., 2014). We abstain from considering completeness in the main result, and directly move from non-satiation to monotonicity.
} 
pursuing a purely algebraic approach. A disadvantage of this level of generality is that we cannot talk about a utility function representing the preference relation.

\section{Results}

Our main result is the equivalence between an axiom of revealed preferences and the rationalization according to $\mathcal{F}$. Denote the composition of $f_{1}, \ldots, f_{n}$ by

$$
\bigcirc_{j=1}^{n} f_{j}=f_{1} \circ f_{2} \circ \ldots \circ f_{n}
$$

denote the downward closure of $B$ by

$$
B^{\geq}=\left\{x: \exists x^{\prime} \in B \text { such that } x^{\prime} \geq x\right\} ;
$$

and denote the strict downward closure of $B$ by

$$
B^{>}=\left\{x: \exists x^{\prime} \in B \text { such that } x^{\prime}>x\right\} .
$$

Our revealed preference axiom follows.

Definition 4. A data set $(\mathcal{B}, C)$ satisfies the Algebraic Axiom of Revealed Preferences (AARP) if for every sequence $x_{1}, \ldots, x_{n}$ such that $x_{j} \in C\left(B_{j}\right)$ for every $j \in\{1, \ldots, n\}$ for some $B_{1}, \ldots, B_{n} \in \mathcal{B}$, and every sequence $f_{1}, \ldots, f_{n-1} \in \mathcal{F}$ such that $f_{j}\left(x_{j+1}\right) \in B_{j}^{\geq}$for every $j \in\{1, \ldots, n-1\}$, we have

$$
\left[\bigodot_{j=1}^{n-1} f_{j}\right]^{-1}\left(x_{1}\right) \notin B_{n}^{>} .
$$

AARP follows the standard logic of Generalized Axiom (GARP) but accounting for the transformations allowed. Recall that GARP states that if there is a sequence of chosen points such that $x_{j+1} \in B_{j}^{\geq}$for every $j \in\{1, \ldots, n-1\}$ then $x_{1} \notin B_{n}^{>}$. Hence, we simply allow for the transformations of every chosen point $x_{2}, \ldots, x_{n}$ but we require to take the inverse of all these transformations in the implication part. Intuitively, using monotonicity and the properties of the group $(\mathcal{F}, \circ)$ we can show that the premise of the axiom implies

$$
\left(\left[\bigcirc_{j=1}^{n-1} f_{j}\right]^{-1}\left(x_{1}\right), x_{n}\right) \in R .
$$


It turns out that this is the only requirement imposed by rationalization.

Theorem 1. A data set $(\mathcal{B}, C)$ is rationalizable with theory $\mathcal{F}$ if and only if it satisfies AARP.

Theorem 1 simply states that rationalization of the data set is equivalent to the provided revealed preference axiom. As we already mentioned, AARP is a ready-made test which can be used with data once the researcher defines the theory which satisfies the algebraic and logical conditions we impose. Further elaborating this idea, in the following section we show that AARP subsumes several axioms from the literature and that Theorem 1 generalizes existing results.

3.1. Applications. In this section we show that classical theories of preferences fit in the framework. Note that for some of the theories we have to introduce some extra structure over the space of alternatives which is not necessary for the general result, but unavoidable once we want to define the particular theory.

\subsubsection{Transitive Preferences.}

Definition 5. A preference relation is said to be transitive if for every $x=s_{1}, \ldots, s_{n}=y \in X$,

$$
\left(s_{j}, s_{j+1}\right) \in R \text { for every } j \in\{1, \ldots, n-1\} \text { implies }(x, y) \in R .
$$

We can define $\mathcal{T}=\{I\}$. Trivially, $(\mathcal{T}, \circ)$ is a group, since it contains a unique element that is the identity function. Moreover, it can be easily seen that $\mathcal{T}$ is the correct theory describing transitive preferences given the definition above. Moreover, AARP in this case is equivalent to GARP.

Definition 6. A data set $(\mathcal{B}, C)$ satisfies the Generalized Axiom of Revealed Preferences (GARP) if for every sequence $x_{1}, \ldots, x_{n}$ such that $x_{j} \in C\left(B_{j}\right)$ for every $j \in\{1, \ldots, n\}$ for some $B_{1}, \ldots, B_{n} \in \mathcal{B}$, if

$$
x_{j+1} \in B_{j}^{\geq} \text {for every } j \in\{1, \ldots, n-1\}
$$

then

$$
x_{1} \notin B_{n}^{>} .
$$


Hence, we can obtain the seminal results of Afriat (1967), Diewert (1973), Varian (1983), and Nishimura et al. (2017) as corollary of our main result.

Corollary 1. A data set $(\mathcal{B}, C)$ is rationalizable with transitive preferences if and only if it satisfies GARP.

3.1.2. Homothetic Preferences. Let $X$ be a vector space over a fully ordered field $A$, and denote by $A_{+}$the subspace of $A$ such that all $\alpha \in A_{i}$ is such that $\alpha \geq 0 .{ }^{4}$

Definition 7. A preference relation is said to be homothetic if for every $x=s_{1}, \ldots, s_{n}=y$ and every $\alpha_{1}, \ldots, \alpha_{n-1} \in A_{+}$, such that $\left(\alpha_{j} s_{j}, \alpha_{j} s_{j+1}\right) \in R$ for every $j \in\{1, \ldots, n-1\}$, we have $(x, y) \in R$.

We can define the $\mathcal{H}=\left\{f(x)=\alpha x: \alpha \in A_{+}\right\}$. It is easy to see that $(\mathcal{H}, \circ)$ is a group. In this case AARP is equivalent to a homothetic axiom of revealed preferences that generalizes the one proposed by Varian (1983), Heufer (2013), and Heufer and Hjertstrand (2019).

Definition 8. A data set $(\mathcal{B}, C)$ satisfies the Homothetic Axiom of Revealed Preferences (HARP) if for every sequence $x_{1}, \ldots, x_{n}$ such that $x_{j} \in C\left(B_{j}\right)$ for every $j \in\{1, \ldots, n\}$ for some $B_{1}, \ldots, B_{n} \in \mathcal{B}$, and every sequence $\alpha_{1} \ldots, \alpha_{n-1} \in A_{+}$such that $\alpha_{j} x_{j+1} \in B_{j}^{\geq}$for every $j \in\{1, \ldots, n-1\}$, we have

$$
\frac{x_{1}}{\prod_{j=1}^{n-1} \alpha_{j}} \notin B_{n}^{>} .
$$

Appealing to Theorem 1 we can immediately obtain the following corollary.

Corollary 2. A data set $(\mathcal{B}, C)$ is rationalizable with homothetic preferences if and only if it satisfies $H A R P$.

3.1.3. Quasilinear Preferences. Let $X$ be a vector space over a fully ordered field $A$. Denote by $e_{i}=(0, \ldots, 0,1,0, \ldots, 0)$ the vector with unique 1 element in the $i$-th place, ${ }^{5}$ since we consider the $i$-th good to be the numeraire.

\footnotetext{
${ }^{4}$ Since $A$ is a field, it contains an element 0 such that $0 * a=0$ for every $a \in A$, with $*$ being the multiplication operator in the field $A$.

${ }^{5}$ Since $A$ is a field, it contains an element 1 such that $1 * a=a$ for every $a \in A$, with $*$ being the multiplication operator in the field $A$.
} 
Definition 9. A preference relation is said to be quasilinear if for every $x=s_{1}, \ldots, s_{n}=y$ and every $\alpha_{1}, \ldots, \alpha_{n-1} \in A$, $\left(s_{j}+\alpha_{j} e_{i}, s_{j+1}+\alpha_{j} e_{i}\right) \in R$ for every $j \in\{1, \ldots, n-1\}$ implies $(x, y) \in R$.

We can define $\mathcal{Q}=\left\{f(x)=x+\alpha e_{i}: \alpha \in A\right\}$. It is easy to see that $(\mathcal{Q}, \circ)$ is a group. In this case, AARP is equivalent to a quasilinear axiom of revealed preferences that generalizes the one proposed by Rochet (1987), Brown and Calsamiglia (2007), and Castillo and Freer (2020).

Definition 10. A data set $(\mathcal{B}, C)$ satisfies the Quasilinear Axiom of Revealed Preferences (QARP) if for every sequence $x_{1}, \ldots, x_{n}$ such that $x_{j} \in C\left(B_{j}\right)$ for every $j \in\{1, \ldots, n\}$ for some $B_{1}, \ldots, B_{n} \in \mathcal{B}$, and every sequence $\alpha_{1}, \ldots, \alpha_{n-1} \in A$ such that $x_{j+1}+\alpha_{j} e_{i} \in B_{j}^{\geq}$for every $j \in\{1, \ldots, n-1\}$, we have

$$
x_{1}-\sum_{j=2}^{n} \alpha_{j} \notin B_{n}^{>} .
$$

Appealing to Theorem 1 we can immediately obtain the following:

Corollary 3. A data set $(\mathcal{B}, C)$ is rationalizable with quasilinear preferences if and only if it satisfies $Q A R P$.

3.2. Completeness. As mentioned before, our rationalization notion does not require existence of the complete relation. We can refine the definition of rationalization in order to include completeness as one of the desired properties for the final relation. A preference relation is said to be complete if for every $x, y \in X$ at least one of the couples $(x, y) \in R$ or $(y, x) \in R$.

Definition 11. A data set $(\mathcal{B}, C)$ is completely rationalizable with theory $\mathcal{F}$ if there is a complete preference relation $R^{*}$ consistent with theory $\mathcal{F}$ such that

$$
(x, y) \in R^{*} \text { for every } x \in C(B) ; y \in B ; B \in \mathcal{B} .
$$

In order to guarantee that complete rationalization is equivalent to AARP, we need to refine the structure of the theory. In particular, let $f \geq f^{\prime}$ if $f(x) \geq f^{\prime}(x)$ for all $x \in X$. A tuple $(\mathcal{F}, \geq)$ is said to be ordered if $f \geq f^{\prime}$ or $f \leq f^{\prime}$ for every $f, f^{\prime} \in \mathcal{F}$. Note that this condition 
is trivially satisfied for transitive, homothetic, and quasilinear theories, since each of these theories depends only on a transformation uniquely determined by a scalar.

Corollary 4. Let $(\mathcal{F}, \geq)$ be ordered. A data set $(\mathcal{B}, C)$ is completely rationalizable with theory $\mathcal{F}$ if and only if it satisfies $A A R P$.

Corollary 4 states that if in addition to $(\mathcal{F}, \circ)$ being a group, $(\mathcal{F}, \geq)$ is fully ordered, then completeness provides no extra empirical content with respect to the basic rationalization. This fact immediately applies to the theories of transitive, homothetic, and quasilinear preferences.

\section{Concluding Remarks}

We provide a comprehensive algebraic approach to revealed preference. We show that if a theory of preferences can be axiomatized in UNCAF form and satisfies some additional algebraic restrictions, then it can be tested using the algebraic axiom of revealed preferences. In addition, we show that the algebraic axiom proposed generalizes existing axioms of revealed preference including axioms for transitive, homothetic and quasilinear preferences. Finally, we show that under some extra conditions, completeness of preferences brings no additional empirical content. The latter result is consistent with the observation of Chambers et al. (2014) who mention that completeness is not an UNCAF axiom and therefore, does not have to be falsifiable.

Our approach provides a fruitful avenue for the future research for several reasons. First, axioms corresponding to different theories over preferences can be immediately deduced from the algebraic axiom (as long as the theories satisfy logical and algebraic conditions). ${ }^{6}$ Second, further restrictions on the family of functions can deliver quite interesting consequences. In particular, if the family of function $(\mathcal{F})$ consists only of linear functions, then the corresponding algebraic axiom can be reduced to a linear programming problem. This is useful because linear programs can be implemented even for extremely large sets of data in a computationally efficient manner, and therefore, it became a desired type of the testing procedure in the revealed preference theory. An

\footnotetext{
${ }^{6}$ For instance, theories that involve independence of preferences can also be incorporated into our framework.
} 
attempt at least to partially characterize the set of theories which can be tested with linear programming is of crucial importance, because it is the same as characterizing the set of theories which can be tested in practice.

\section{Appendix A. Proofs}

The proof proceeds via the construction of a supplementary object we refer to as the sequential closure, which is a function over preference relations. The sequential closure operationalizes the notion of a theory. Note that throughout the paper we maintain the assumption that $(\mathcal{F}, \circ)$ is a group.

Definition 12. $F: \mathcal{R} \rightarrow \mathcal{R}$ is said to be a sequential closure, $(x, y) \in F(R)$ if and only if there are finite sequences

$$
x=s_{1}, \ldots, s_{n}=y \in X \text { and } f_{1}, \ldots, f_{n-1} \in \mathcal{F}
$$

such that

$$
\forall j \in\{1, \ldots, n-1\},\left(f_{j}\left(s_{j}\right), f_{j}\left(s_{j+1}\right)\right) \in R .
$$

It is readily seen that a monotone preference relation $R$ is a fixed point of $F$, that is $R=F(R)$, if and only if it is consistent with the theory $\mathcal{F}$. In particular, if $R=F(R)$, it follows immediately from the definition of consistency that $R$ is consistent with $\mathcal{F}$. If $R$ is consistent with $\mathcal{F}$, it follows immediately from the definition of sequential closure that $F(R) \subset R$, and using the fact that $\mathcal{F}$ is closed under inversion, $R \subset F(R)$.

The following result clarifies the relationship between $F$ and $\mathcal{F}$.

Lemma 1. Let $F: \mathcal{R} \rightarrow \mathcal{R}$ be a sequential closure. Then $(x, y) \in F(R)$ if and only if $(f(x), f(y)) \in F(R)$ for every $f \in \mathcal{F}$.

Proof. Let $(x, y) \in F(R)$, then there are sequences $x=s_{1}, \ldots, s_{n}=$ $y \in X$ and $f_{1}, \ldots, f_{n-1} \in \mathcal{F}$ such that

$$
\left(f_{j}\left(s_{j}\right), f_{j}\left(s_{j+1}\right)\right) \in R \text { for every } j \in\{1, \ldots, n-1\} .
$$

Consider any $f \in \mathcal{F}$. To show that $(f(x), f(y)) \in F(R)$, we construct the following sequences:

$$
f(x)=\hat{s}_{1}=f\left(s_{1}\right), \hat{s}_{2}=f\left(s_{2}\right), \ldots, \hat{s}_{n}=f\left(s_{n}\right)=f(y)
$$


and

$$
\hat{f}_{1}=\left[f_{1} \circ f^{-1}\right], \hat{f}_{2}=\left[f_{2} \circ f^{-1}\right], \ldots, \hat{f}_{n-1}=\left[f_{n-1} \circ f^{-1}\right] .
$$

Note that $\mathcal{F}$ is closed under inversion, so $\hat{f}_{j} \in \mathcal{F}$ for $j \in\{1, \ldots, n-1\}$. Moreover,

$$
\begin{array}{r}
\left(\hat{f}_{j}\left(\hat{s}_{j}\right), \hat{f}_{j}\left(\hat{s}_{j+1}\right)\right)=\left(f_{1}\left(f^{-1}\left(f\left(s_{j}\right)\right)\right), f_{1}\left(f^{-1}\left(f\left(s_{j+1}\right)\right)\right)\right)= \\
\quad=\left(f_{j}\left(s_{j}\right), f_{j}\left(s_{j+1}\right)\right) \in R \text { for every } j \in\{1, \ldots, n-1\} .
\end{array}
$$

Using the definition of sequential closure, we get $(f(x), f(y)) \in F(R)$. The inverse causality follows from a similar argument.

In order to develop our argument we need to prove that the sequential closure exhibits several convenient properties.

Lemma 2. If $F: \mathcal{R} \rightarrow \mathcal{R}$ is a sequential closure, then it is

- increasing: for all $R \in \mathcal{R}, R \subseteq F(R)$,

- monotonic: for all $R, R^{\prime} \in \mathcal{R}$, if $R \subseteq R^{\prime}$, then $F(R) \subseteq F\left(R^{\prime}\right)$,

- idempotent: if for all $R \in \mathcal{R}, F(F(R))=F(R)$,

- algebraic: for all $R \in \mathcal{R}$ and all $(x, y) \in F(R)$, there is a finite relation $R^{\prime} \subseteq R$ such that $(x, y) \in F\left(R^{\prime}\right)$,

- and induces transitivity: $F(R)=T(F(R))$.

Proof. $F$ is increasing.

Recall that $I \in \mathcal{F}$. Hence, for every $(x, y) \in R$ there is a sequence $x=s_{1}, s_{2}=y$ and $f_{1}=I$, such that $I(x, y) \in R$, and therefore $(x, y) \in F(R)$.

\section{$F$ is monotonic.}

Take $(x, y) \in F(R)$. Then there are sequences $x=s_{1}, \ldots, s_{n}=y \in$ $X$ and $f_{1}, \ldots, f_{n-1} \in \mathcal{F}$ such that $f_{j}\left(s_{j}, s_{j+1}\right) \in R \subseteq R^{\prime}$. Since $f_{j}\left(s_{j}, s_{j+1}\right) \in R^{\prime}$ for every $j \in\{1, \ldots, n-1\}$, we have $(x, y) \in F\left(R^{\prime}\right)$.

\section{$F$ is idempotent.}

Note that from the fact that $F$ is increasing we know that $F(R) \subseteq$ $F(F(R))$. Hence, we are left to show that $F(F(R)) \subseteq F(R)$. Consider $(x, y) \in F(F(R))$, and note that that by the definition of sequential closure there are sequences $x=s_{1}, \ldots, s_{n}=y$ and $f_{1}, \ldots, f_{n-1}$ such 
that

$$
\left(f_{j}\left(s_{j}\right), f_{j}\left(s_{j+1}\right)\right) \in F(R) \text { for every } j \in\{1, \ldots, n-1\} .
$$

Using Lemma 1, we can substitute each $f_{j}$ with $I$, giving

$$
\left(s_{j}, s_{j+1}\right) \in F(R) .
$$

But then there are sequences $s_{j}=s_{1}^{j}, \ldots, s_{n_{j}}^{j}$ and $f_{1}^{j}, \ldots, f_{n_{j-1}}^{j} \in \mathcal{F}$ such that

$$
\left(f_{k}^{j}\left(s_{k}^{j}\right), f_{k}^{j}\left(s_{k+1}^{j}\right)\right) \in R \text { for every } k \in\left\{1, \ldots, n_{j}-1\right\} .
$$

Merging all such sequences in one, it follows that $(x, y) \in F(R)$. Therefore, $F(F(R)) \subseteq F(R)$.

\section{$F$ is algebraic.}

Consider a relation $R$ and an element $(x, y) \in F(R)$, then there are sequences $x=s_{1}, \ldots, s_{n}$ and $f_{1}, \ldots, f_{n-1}$ such that $f_{j}\left(s_{j}, s_{j+1}\right) \in R$. Let $D=\left\{s_{1}, \ldots, s_{n}\right\}$ and let $R^{\prime}=R \cap(D \times D)$. Then, $(x, y) \in F\left(R^{\prime}\right)$ and $R^{\prime}$ is finite by definition.

\section{$F$ induces transitivity.}

Using $I \in \mathcal{F}$, it is easy to check that $T(R) \subseteq F(R)$. Given that $T$ is also increasing and monotonic $(\operatorname{since}(\mathcal{T}, \circ)$ is trivially a group), then (1) $F(R) \subseteq T(F(R))$, and idempotence of $F$ implies $(2) T(F(R)) \subseteq$ $F(F(R))=F(R)$. Hence, $T(F(R))=F(R)$.

Next we need to introduce the notion of extension of one preference relation by another. Let $R \preceq R^{\prime}$ ( $R^{\prime}$ is an extension of $R$ ) if $R \subseteq R^{\prime}$ and $P(R) \subseteq P\left(R^{\prime}\right)$. In addition, we introduce the operationalizable version of the same definition.

Lemma 3 (Demuynck (2009); Freer and Martinelli (2019)). Let $R \subseteq$ $R^{\prime} . R \preceq R^{\prime}$ if and only if $P^{-1}(R) \cap R^{\prime}=\emptyset$.

Finally, for a given data set $(\mathcal{B}, C)$, we introduce the revealed preference relation denoted by $R_{E}$, such that $(x, y) \in R_{E}$ if $x \in C(B)$ and $y \in B$ for some $B \in \mathcal{B}$ or if $x=y$. (Since $R_{E}$ is reflexive, it is a preference relation.) 
A.1. Proof of Theorem 1. Recall that a monotone preference relation $R$ is consistent with $\mathcal{F}$ if and only if $R$ it is a fixed point of $F$, that is $F(R)=R$.

Lemma 4. A data set $(\mathcal{B}, C)$ is rationalizable if and only if $\geq \preceq$ $F\left(R_{E} \cup \geq\right)$.

Proof. $(\Rightarrow)$ On the contrary, assume $(\mathcal{B}, C)$ is rationalizable but it is not the case that $\geq \preceq F\left(R_{E} \cup \geq\right)$. Appealing to Lemma 3, then there exists some $(z, w)$ such that $(w, z) \in>$ and $(z, w) \in F\left(R_{E} \cup \geq\right)$. Since $(\mathcal{B}, C)$ is rationalizable, there is a monotone preference relation $R^{*}$ that is consistent with theory $\mathcal{F}$ and such that

$$
\left(x_{j}, y\right) \in R^{*} \text { for every } x_{j} \in C\left(B_{j}\right) ; y \in B_{j} \text { for every } B_{j} \in \mathcal{B} .
$$

Hence, $R_{E} \cup \geq \subseteq R^{*}$ and $\geq \preceq R^{*}$. Moreover, since $R^{*}$ is consistent with theory $\mathcal{F}$, it is a fixed point of $F$, i.e. $F\left(R^{*}\right)=R^{*}$. Therefore, given that $F$ is monotonic, we have $F\left(R_{E} \cup \geq\right) \subseteq F\left(R^{*}\right)=R^{*}$. Hence, $(z, w) \in R^{*}$ implying $\geq \cap R^{*} \neq \emptyset$, which contradicts the fact that $R^{*}$ is monotone.

$(\Leftarrow)$ Let $\tilde{R}=F\left(R_{E} \cup \geq\right)$. $\tilde{R}$ is monotone since $\geq \preceq F\left(R_{E} \cup \geq\right)$. Since $F$ is increasing, $R_{E} \subseteq \tilde{R}$ that is $(x, y) \in \tilde{R}$ if $x \in C(B)$ and $y \in B$ for every $B \in \mathcal{B}$. Finally, since $F$ is idempotent,

$$
F(\tilde{R})=F\left(F\left(R_{E} \cup \geq\right)\right)=F\left(R_{E} \cup \geq\right)=\tilde{R},
$$

that is $\tilde{R}$ is a fixed point of $F$. Therefore, $\tilde{R}$ is consistent with the theory.

Next, we show that AARP is equivalent to $\geq \preceq F\left(R_{E} \cup \geq\right)$. Before we proceed, we introduce two auxiliary results. The first deals with the nature of the fixed points of the sequential closure, while the second deals with the nature of the shortest sequence adding any $(x, y) \in X^{2}$ to $F\left(R_{E} \cup \geq\right)$.

Lemma 5. Let $F: \mathcal{R} \rightarrow \mathcal{R}$ be a sequential closure and let $R$ be a fixed point of $F$. Then $(x, y) \in P(R)$ implies $(f(x), f(y)) \in P(R)$ for every $f \in \mathcal{F}$.

Proof. From Lemma 1, $(x, y) \in P(R) \subseteq R$ implies $(f(x), f(y)) \in R=$ $F(R)$ for every $f \in \mathcal{F}$. To see this, note that $(\mathcal{F}, \circ)$ is a group and 
therefore, $f^{-1} \in \mathcal{F}$. Hence, by construction of the sequential closure we can infer that there are sequences $s_{1}=f(x), s_{2}=f(y)$ and $f_{1}=$ $f^{-1}$ such that $(x, y)=\left(\left[f^{-1} \circ f\right](x),\left[f^{-1} \circ f\right](y)\right) \in R$ and therefore $(f(x), f(y)) \in F(R)=R$ since $R$ is a fixed point of $F$.

Next we show that $(f(y), f(x)) \notin R$, completing the proof. On the contrary, suppose $(f(y), f(x)) \in R$. We can construct the sequence $y=s_{1}$ and $s_{2}=x$, and let $f_{1}=f$ so that $(f(y), f(x)) \in R$ and hence $(y, x) \in F(R)=R$, contradicting $(x, y) \in P(R)$.

Lemma 6. Let $F: \mathcal{R} \rightarrow \mathcal{R}$ be a sequential closure and $\left(s_{1}, \ldots, s_{n}\right)$ and $\left(f_{1}, \ldots, f_{n-1}\right)$ be any of the shortest pairs of sequences adding $(x, y) \in X \times X$ to $F\left(R_{E} \cup \geq\right)$. Then, for every $j \in\{1, \ldots, n-2\}$, $\left(f_{j}\left(s_{j}\right), f_{j}\left(s_{j+1}\right)\right) \in \geq$ implies $\left(f_{j+1}\left(s_{j+1}\right), f_{j+1}\left(s_{j+2}\right)\right) \in R_{E}$.

Proof. Assume $\left(f_{j}\left(s_{j}\right), f_{j}\left(s_{j+1}\right)\right) \in \geq$ and $\left(f_{j+1}\left(s_{j+1}\right), f_{j+1}\left(s_{j+2}\right)\right) \notin R_{E}$ for some $1 \leq j \leq n-3$. It follows that $\left(f_{j+1}\left(s_{j+1}\right), f_{j+1}\left(s_{j+2}\right)\right) \in \geq$. From $\geq=F(\geq)$, we have $\left(s_{j}, s_{j+1}\right) \in \geq$ and $\left(s_{j+1}, s_{j+2}\right) \in \geq$. Since $\geq$ is transitive, we can shorten the sequence by omitting $s_{j+1}$ and using $\left(s_{j}, s_{j+2}\right) \in \geq$ and $\left(f_{j+2}\left(s_{j+2}\right), f_{j+2}\left(s_{j+3}\right)\right) \in R_{E} \cup \geq$. This contradicts the fact that the original sequence is shortest.

Using the results above we show that AARP is equivalent to the consistency of revealed preference relation.

Lemma 7. Let $F$ be a sequential closure. A data set satisfies AARP if and only if $>^{-1} \cap F\left(R_{E} \cup \geq\right)=\emptyset$.

Proof. $(\Leftarrow)$ Assume on the contrary that there is a violation of AARP. That is, there is a sequence $x_{1}, \ldots, x_{n}$ such that $x_{j} \in C\left(B_{j}\right)$ for every $j \in\{1, \ldots, n\}$ for some $B_{1}, \ldots, B_{n} \in \mathcal{B}$, and a sequence $f_{1}, \ldots, f_{n-1} \in$ $\mathcal{F}$ such that $f_{j}\left(x_{j+1}\right) \in B_{j}^{\geq}$for every $j \in\{1, \ldots, n-1\}$, but

$$
\left[\bigcirc_{j=1}^{n-1} f_{j}\right]^{-1}\left(x_{1}\right) \in B_{n}^{>} .
$$

To proceed with the proof, note that $f_{j}\left(x_{j+1}\right) \in B_{j}^{\geq}$implies that there is $y_{j}$ such that $\left(x_{j}, y_{j}\right) \in R_{E}$ and $\left(y_{j}, f_{j}\left(x_{j+1}\right)\right) \in \geq$. 
We construct a sequence $s_{1}, \ldots, s_{2 n-1}$ as follows. Let

$$
s_{2 j-1}=\left[\bigcirc_{k=j}^{n-1} f_{k}\right]^{-1}\left(x_{j}\right) \text { and } s_{2 j}=\left[\bigcirc_{k=j}^{n-1} f_{k}\right]^{-1}\left(y_{j}\right) \text {, }
$$

for $j \in\{1, \ldots, n-1\}$, and let $s_{2 n-1}=x_{n}$. Next, we construct a sequence of functions $\hat{f}_{1}, \ldots, \hat{f}_{2 n-2}$, where

$$
\hat{f}_{2 j-1}(x)=\hat{f}_{2 j}(x)=\left[\bigcirc_{k=j}^{n-1} f_{k}\right](x)
$$

for $j \in\{1, \ldots, n-1\}$.

For any $j \in\{1, \ldots, n-1\}$, consider the triple $s_{2 j-1}, s_{2 j}, s_{2 j+1}$. We have

$$
\begin{aligned}
& \left(\hat{f}_{2 j-1}\left(s_{2 j-1}\right), \hat{f}_{2 j-1}\left(s_{2 j}\right)\right) \\
& \quad=\left(\left[\bigcirc_{k=j}^{n-1} f_{k}\right] \circ\left[\bigcirc_{k=j}^{n-1} f_{k}\right]^{-1}\left(x_{j}\right),\left[\bigcirc_{k=j}^{n-1} f_{k}\right] \circ\left[\bigcirc_{k=j}^{n-1} f_{k}\right]^{-1}\left(y_{j}\right)\right) \\
& =\left(x_{j}, y_{j}\right) \in R_{E},
\end{aligned}
$$

and

$$
\begin{aligned}
& \left(\hat{f}_{2 j}\left(s_{2 j}\right), \hat{f}_{2 j}\left(s_{2 j+1}\right)\right) \\
& \quad=\left(\left[\bigcirc_{k=j}^{n-1} f_{k}\right] \circ\left[\bigcirc_{k=j}^{n-1} f_{k}\right]^{-1}\left(y_{j}\right),\left[\bigcirc_{k=j}^{n-1} f_{k}\right] \circ\left[\bigcirc_{k=j+1}^{n-1} f_{k}\right]^{-1}\left(x_{j+1}\right)\right) \\
& \quad=\left(y_{j}, f_{j}\left(x_{j+1}\right)\right) \in \geq .
\end{aligned}
$$

Since $(\mathcal{F}, \circ)$ is a group we have $\hat{f}_{2 j-1}(x), \hat{f}_{2 j}(x) \in \mathcal{F}$, which in turn implies $\left(s_{1}, s_{2 n-1}\right)=\left(s_{1}, x_{n}\right) \in F\left(R_{E} \cup \geq\right)$.

Using the premise

$$
s_{1}=\left[\bigcirc_{k=1}^{n-1} f_{k}\right]^{-1}\left(x_{1}\right) \in B_{n}^{>},
$$

there is $y_{n} \in B_{n}$ such that $\left(y_{n}, s_{1}\right) \in>$. Moreover, $\left(x_{n}, y_{n}\right) \in R_{E}$ by construction of the revealed preference relation. Given that $F(R)$ is transitivity inducing, $\left(s_{1}, y_{n}\right) \in F\left(R_{E} \cup \geq\right)$. But then

$$
\left(s_{1}, y_{n}\right) \in>^{-1} \cap F\left(R_{E} \cup \geq\right),
$$

implying $>^{-1} \cap F\left(R_{E} \cup \geq\right) \neq \emptyset$. 
$(\Rightarrow)$ On the contrary, assume that there is a violation of consistency, i.e. there is $(z, w) \in>^{-1} \cap F\left(R_{E} \cup \geq\right)$. Consider any shortest sequence adding $(z, w)$ to $F\left(R_{E} \cup \geq\right)$, that is $z=s_{1}, \ldots, s_{n}=w$ such that

$$
\left(f_{j}\left(s_{j}\right), f_{j}\left(s_{j+1}\right)\right) \in R_{E} \cup \geq \text { for every } j \in\{1, \ldots, n-1\} \text { and } f_{j} \in \mathcal{F} \text {. }
$$

By definition, for every $j$ such that $\left(f_{j}\left(s_{j}\right), f_{j}\left(s_{j+1}\right)\right) \in R_{E}$ there is some $B_{j} \in \mathcal{B}$ such that $f_{j}\left(s_{j}\right) \in C\left(B_{j}\right)$ and $f_{j}\left(s_{j+1}\right) \in B_{j}$. For every $j$ such that $\left(f_{j}\left(s_{j}\right), f_{j}\left(s_{j+1}\right)\right) \in R_{E}$, define

$$
m_{j}=\mid\left\{s_{l}:\left(f_{l}\left(s_{l}\right), f_{l}\left(s_{l+1}\right)\right) \notin R_{E} \text { and } l<j\right\} \mid .
$$

We now build a sequence $x_{1}, \ldots, x_{m}$ and a sequence $\hat{B}_{1}, \ldots, \hat{B}_{m}$ where $m=n-m_{n}$ and

$$
x_{j-m_{j}}=f_{j}\left(s_{j}\right) \text { and } \hat{B}_{j-m_{j}}=B_{j}
$$

for every $j \in\{1, \ldots, n-1\}$ such that $\left(f_{j}\left(s_{j}\right), f_{j}\left(s_{j+1}\right)\right) \in R_{E}$, and $x_{m}=f_{n-1}\left(s_{n}\right)$ and $\hat{B}_{m}=\left\{f_{n-1}\left(s_{n}\right)\right\}$.

Similarly, let $\tilde{f}_{j-m_{j}}=f_{j}$ for every $j \in\{1, \ldots, n-1\}$ such that $\left(f_{j}\left(s_{j}\right), f_{j}\left(s_{j+1}\right)\right) \in R_{E}$ and $\tilde{f}_{m}=I$ (the identity function), and define

$$
\hat{f}_{k}=\tilde{f}_{k} \circ \tilde{f}_{k+1}^{-1}
$$

for $k \in\{1, \ldots, m-1\}$.

Note that for every $k \in\{1, \ldots, m\}$ we have $x_{k} \in C\left(\hat{B}_{k}\right)$. We claim $\hat{f}_{k}\left(x_{k+1}\right) \in B_{k}^{\geq}$for every $k \in\{1, \ldots, m-1\}$. To see this, note that given the structure of the original sequence described in Lemma 6 , either

(1) $x_{k}=f_{j}\left(s_{j}\right)$ and $x_{k+1}=f_{j+1}\left(s_{j+1}\right)$ for some $j \in\{1, \ldots, n-1\}$, with $\left(f_{j}\left(s_{j}\right), f_{j}\left(s_{j+1}\right)\right) \in R_{E}$, or

(2) $x_{k}=f_{j}\left(s_{j}\right)$ and $x_{k+1}=f_{j+2}\left(s_{j+2}\right)$ for some $j \in\{1, \ldots, n-2\}$, with $\left(f_{j}\left(s_{j}\right), f_{j}\left(s_{j+1}\right)\right) \in R_{E}$ and $\left(f_{j+1}\left(s_{j+1}\right), f_{j+1}\left(s_{j+2}\right)\right) \in \geq$.

In case (1), the result is immediate since

$$
\hat{f}_{k}\left(x_{k+1}\right)=\left[f_{j} \circ f_{j+1}^{-1}\right]\left(f_{j+1}\left(s_{j+1}\right)\right)=f_{j}\left(s_{j+1}\right) \in B_{j}=\hat{B}_{k} .
$$

In case (2), we can appeal to $\geq=F(\geq)$ to show that $f_{j}\left(s_{j+1}\right) \geq$ $f_{j}\left(s_{j+2}\right)$. Hence,

$$
\hat{f}_{k}\left(x_{k+1}\right)=\left[f_{j} \circ f_{j+2}^{-1}\right]\left(f_{j+2}\left(s_{j+2}\right)\right)=f_{j}\left(s_{j+2}\right) \in B_{j}^{\geq}=B_{k}^{\geq} .
$$


Finally, note that

$$
\begin{aligned}
{\left[\bigcirc_{k=1}^{m-1} \hat{f}_{k}\right]^{-1} } & =\left[\left[\tilde{f}_{1} \circ \tilde{f}_{2}^{-1}\right] \circ\left[\tilde{f}_{2} \circ \tilde{f}_{3}^{-1}\right] \circ \cdots \circ\left[\tilde{f}_{m-2} \circ \tilde{f}_{m-1}^{-1}\right]\right]^{-1}= \\
& =\left[\tilde{f}_{1} \circ \tilde{f}_{m}^{-1}\right]^{-1}=\tilde{f}_{m} \circ \tilde{f}_{1}^{-1} .
\end{aligned}
$$

Since $\left(f_{n-1}\left(s_{n-1}\right), f_{n-1}\left(s_{n}\right)\right) \in R_{E}$, we have $\tilde{f}_{m-1}=f_{n-1}$. Given lemma 6(1), either

$$
\text { (1) } \tilde{f}_{1}=f_{1} \text {, or }(2)\left(f_{1}\left(s_{1}\right), f_{1}\left(s_{2}\right)\right) \in \geq \text { and } \tilde{f}_{1}=f_{2} \text {. }
$$

We build a contradiction to AARP for each case. In case (1), we have

$$
\left[\bigcirc_{k=1}^{m-1} \hat{f}_{k}^{-1}\right]\left(x_{1}\right)=f_{n-1}(z)
$$

Using $\geq=F(\geq)$ and $(w, z) \in>$, we have $f_{n-1}(w)>f_{n-1}(z)$. Recall that $\hat{B}_{m}=\left\{f_{n-1}\left(s_{n}\right)\right\}=\left\{f_{n-1}(w)\right\}$. Thus,

$$
\left[\bigcirc_{k=1}^{m-1} \hat{f}_{k}^{-1}\right]\left(x_{1}\right) \in \hat{B}_{m}^{>}
$$

a contradiction with AARP.

In case (2), we have

$$
\left[\bigcirc_{k=1}^{m-1} \hat{f}_{k}^{-1}\right]\left(x_{1}\right)=f_{n-1}\left(s_{2}\right) \quad \text { and } \quad\left(f_{1}\left(s_{1}\right), f_{1}\left(s_{2}\right)\right) \in \geq .
$$

Using $\geq=F(\geq)$ and $(w, z) \in>$, we have

$$
f_{n-1}(w)>f_{n-1}(z)=f_{n-1}\left(s_{1}\right) \geq f_{n-1}\left(s_{2}\right) .
$$

Thus,

$$
\left[\bigcirc_{k=1}^{m-1} \hat{f}_{k}^{-1}\right]\left(x_{1}\right) \in \hat{B}_{m}^{>}
$$

a contradiction with AARP.

Proof of Theorem 1. From Lemma 3, $\preceq \preceq F\left(R_{E} \cup \geq\right)$ if and only if $P^{-1}(R) \cap F\left(R_{E} \cup \geq\right)=\emptyset$. From Lemma 4 , a data set $(\mathcal{B}, C)$ is rationalizable if and only if $\geq \preceq F\left(R_{E} \cup \geq\right)$. Finally, from Lemma 7, $P^{-1}(R) \cap F\left(R_{E} \cup \geq\right)=\emptyset$ if and only if AARP holds. 
A.2. Proof of Corollary 4. Note that the proof of Theorem 1 shows that AARP is equivalent to the partial rationalization of the data. Equivalently, there is a monotone preference relation $R^{*}$ that is a fixed point of $F$ and that subsumes the revealed preference relation. To prove the corollary, we need to show that there is a complete preference relation $\hat{R}$ that is a fixed point of $F$ and $R^{*} \preceq \hat{R}$. For this purpose we use Demuynck (2009) extension theorem, which requires us to introduce an additional property. Let $N(R)=(X \times X) \backslash\left(R \cup R^{-1}\right)$ be the incomparable part of $R$.

Definition 13. A function $F: \mathcal{R} \rightarrow \mathcal{R}$ is said to be weakly expansive if for any $\geq \preceq R=F(R)$ and $N(R) \neq \emptyset$ there is a nonempty $S \subseteq N(R)$ such that $\geq \preceq F(R \cup S)$ and $R \cup S \preceq F(R \cup S)$.

If $F$ is weakly expansive, we can appeal to Demuynck (2009) extension theorem.

Lemma 8 (Demuynck (2009) Extension Theorem). Let $F: \mathcal{R} \rightarrow \mathcal{R}$ be a weakly expansive, increasing, monotonic, idempotent, and algebraic function. There is a complete, fixed point extension of $R$ if and only if $R \preceq F\left(R^{\prime}\right)$ for some $R^{\prime} \supseteq R .^{7}$

Recall that a relation is consistent with the theory if and only if it is a fixed point relation. Hence, a complete, fixed point extension is the object of interest.

It remains to be shown that any fully ordered theory generates a weakly expansive closure.

Lemma 9. If a function $F: \mathcal{R} \rightarrow \mathcal{R}$ is an ordered sequential closure, then it is weakly expansive.

Proof. Take some $R$ such that $\geq \preceq R=F(R)$ and some $(x, y) \in N(R)$, and let $R^{\prime}=R \cup\{(x, y)\}$. From Lemma 3, it is enough to show that $>^{-1} \cap F\left(R^{\prime}\right)=\emptyset$ and $P^{-1}\left(R^{\prime}\right) \cap F\left(R^{\prime}\right)=\emptyset$. On the contrary, assume

$$
(z, w) \in\left(>^{-1} \cup P^{-1}\left(R^{\prime}\right)\right) \cap F\left(R^{\prime}\right) .
$$

Since $(z, w) \in F\left(R^{\prime}\right)$, there are sequences $z=s_{1}, \ldots, s_{n}=w$ and $f_{1}, \ldots, f_{n-1}$ such that $\left(f_{j}\left(s_{j}\right), f_{j}\left(s_{j+1}\right)\right) \in R^{\prime}$. We consider any shortest

\footnotetext{
${ }^{7}$ We have modified the statement of the theorem to suit our notation, but this is equivalent to theorem 2 in Demuynck (2009); see also Freer and Martinelli (2019).
} 
sequence. Next, we prove two properties of such sequences.

Claim: $\exists k \leq n-1$ such that $\left(f_{k}\left(s_{k}\right), f_{k}\left(s_{k+1}\right)\right)=(x, y)$.

On the contrary, assume there is no such $k$. Then, $\left(f_{j}\left(s_{j}\right), f_{j}\left(s_{j+1}\right)\right) \in R$ for all $j \leq n-1$, since $R^{\prime} \backslash R=\{(x, y)\}$. The construction of the sequence implies then that $(z, w) \in F(R)$. This is a contradiction since $(z, w) \in>^{-1}$ implies $(w, z) \in>\subseteq P(R)=P(F(R))$, and similarly $(z, w) \in P^{-1}(R)$ implies $(w, z) \in P(R)=P(F(R))$.

Claim: $(x, y)$ appears in every shortest sequence only once.

Assume on the contrary that $(x, y)$ enters the sequence more than once; that is, there are $k<l<n$ such that $\left(f_{k}\left(s_{k}\right), f_{k}\left(s_{k+1}\right)\right)=(x, y)=$ $\left(f_{l}\left(s_{l}\right), f_{l}\left(s_{l+1}\right)\right)$. Without loss of generality, suppose these are the first and the second time that $(x, y)$ enters into the sequence. Recall that we consider a set of ordered functions. Hence, either $f_{k}>f_{l}$ or $f_{k} \leq f_{l}$. We consider the two cases separately.

Case 1: $f_{k} \leq f_{l}$. Since $f_{k} \leq f_{l}$ means that $f_{k}(x) \leq f_{l}(x)$ for every $x \in X$, then $f_{k}^{-1} \geq f_{l}^{-1}$. The latter fact implies $\left(s_{k+1}, s_{l+1}\right) \in \geq \subseteq R^{\prime}$. Hence, if $l>k+1$, the sequence can be shortened by eliminating $s_{l}$. That is, if the original sequence is such that

$$
\ldots,\left(f_{k}\left(s_{k}\right), f_{k}\left(s_{k+1}\right)\right)=(x, y) \in R^{\prime}, \ldots,\left(f_{l}\left(s_{l}\right), f_{l}\left(s_{l+1}\right)\right)=(x, y) \in R^{\prime}, \ldots
$$

we can use instead

$$
\ldots,\left(f_{k}\left(s_{k}\right), f_{k}\left(s_{k+1}\right)\right)=(x, y) \in R^{\prime},\left(s_{k+1}, s_{l+1}\right) \in \geq \subseteq R^{\prime}, \ldots
$$

This contradicts the assumption that the original sequence is one of the shortest.

If instead $l=k+1$, using $f_{k}\left(s_{k}\right)=f_{l}\left(s_{l}\right)=x$ and $f_{k}\left(s_{k+1}\right)=$ $f_{l}\left(s_{l+1}\right)=y$, we get $s_{k} \geq s_{l}=s_{k+1} \geq s_{l+1}$. Thus, $\left(s_{k}, s_{l+1}\right) \in \geq \subseteq R^{\prime}$, and the initial sequence can be shortened by eliminating $s_{l}=s_{k+1}$.

Case 2: $f_{k}>f_{l}$. In this case, we have $f_{k}^{-1}<f_{l}^{-1}$, which in turn implies $\left(s_{l}, s_{k}\right) \in>\subset R$. Since $k, l$ correspond to the first and the second time that $(x, y)$ appears in the sequence, we know that for every 
$h$ such that $l<h<k,\left(f_{h}\left(s_{h}\right), f_{h}\left(s_{h+1}\right)\right) \neq(x, y)$ and hence by construction $\left(f_{h}\left(s_{h}\right), f_{h}\left(s_{h+1}\right)\right) \in R=F(R)$. Combining two facts above and taking into account that $F$ is transitivity inducing, we obtain that $\left(s_{k+1}, s_{k}\right) \in R=F(R)$. Therefore, $(y, x)=\left(f_{k}\left(s_{k+1}\right), f_{k}\left(s_{k}\right)\right) \in R$, which contradicts the premise that $(x, y) \in N(R)$.

Given the two claims above we can complete the proof of the Lemma by considering one of shortest sequences with $\left(f_{k}\left(s_{k}\right), f_{k}\left(s_{k+1}\right)\right)=(x, y)$ and with the rest of the elements of the sequence being such that $\left(f_{j}\left(s_{j}\right), f_{j}\left(s_{j+1}\right)\right) \in R$ for every $j \neq k$. Hence, $\left(s_{k+1}, w\right) ;\left(z, s_{k}\right) \in R=$ $F(R)$. Given that either $(w, z) \in>\subseteq R$ or $(w, z) \in P(R) \subset R$, we have $\left(s_{k}, s_{k+1}\right) \in R=F(R)$. Therefore, $(x, y)=\left(f_{k}\left(s_{k}\right), f_{k}\left(s_{k+1}\right)\right) \in$ $R=F(R)$, which contradicts the premise that $(x, y) \in N(R)$. 


\section{REFERENCES}

Sidney N. Afriat. The construction of utility functions from expenditure data. International Economic Review, 8(1):67-77, 1967.

Donald J. Brown and Caterina Calsamiglia. The nonparametric approach to applied welfare analysis. Economic Theory, 31(1):183-188, 2007.

Marco Castillo and Mikhail Freer. A general revealed preference test of quasi-linear prefer- ences: Theory and experiments. Working Paper, Texas A \& M and George Mason University, 2020.

Christopher P. Chambers and Federico Echenique. Revealed Preference Theory. Cambridge University Press, 2016.

Christopher P. Chambers, Federico Echenique, and Eran Shmaya. The axiomatic structure of empirical content. American Economic Review, 104(8):2303-2219, 2014.

Thomas Demuynck. A general extension result with applications to convexity, homotheticity and monotonicity. Mathematical Social Sciences, 57(1):96-109, 2009.

W. Erwin Diewert. Afriat and revealed preference theory. Review of Economic Studies, 40(3):419-425, 1973.

John Duggan. A general extension theorem for binary relations. Journal of Economic Theory, 86(1):1-16, 1999.

Francoise Forges and Enrico Minelli. Afriat's theorem for general budget sets. Journal of Economic Theory, 144(1):135-145, 2009.

Mikhail Freer and César Martinelli. A representation theorem for general revealed preference. Working Paper, George Mason University, 2019.

Jan Heufer. Testing revealed preferences for homotheticity with twogood experiments. Experimental Economics, 16(1):114-124, 2013.

Jan Heufer and Per Hjertstrand. Homothetic preferences revealed. Journal of Economic Behavior \& Organization, 157:602-614, 2019.

Hiroki Nishimura, Efe A. Ok, and John K.-H. Quah. A comprehensive approach to revealed preference theory. American Economic Review, 107(4):1239-1263, 2017.

Matthew Polisson, John K-H Quah, and Ludovic Renou. Revealed preferences over risk and uncertainty. Institute for Fiscal Studies 
Working Paper W15/25, 2015.

Marcel K. Richter. Revealed preference theory. Econometrica, 34(3): 635-645, 1966.

Jean-Charles Rochet. A necessary and sufficient condition for rationalizability in a quasi-linear context. Journal of Mathematical Economics, 16(2):191-200, 1987.

Paul A. Samuelson. A note on the pure theory of consumer's behaviour. Economica, 5(17):61-71, 1938.

Kotaro Suzumura. Remarks on the theory of collective choice. Economica, pages 381-390, 1976.

Edward Szpilrajn. Sur l'extension de l'ordre partiel. Fundamenta mathematicae, 1(16):386-389, 1930.

Hal R. Varian. Non-parametric tests of consumer behaviour. Review of Economic Studies, 50(1):99-110, 1983. 